Investigating factors affecting the body temperature of dogs competing in cross country (canicross) races in the $\mathrm{UK}$.

Anne Carter BSc (Hons) MSc PhD FHEA MRSB (Corresponding author)

Senior Lecturer in Animal Science,

School of Animal, Rural and Environmental Science,

Nottingham Trent University,

Brackenhurst,

Southwell, Nottinghamshire

NG25 OQF

Tel: 01158485281

Email:anne.carter@ntu.ac.uk

Emily J. Hall MA VetMB AFHEA MRSB MRCVS

Lecturer in Veterinary Nursing Science,

School of Animal, Rural and Environmental Science,

Nottingham Trent University,

Brackenhurst,

Southwell, Nottinghamshire

NG25 OQF

Tel: 01158485303

Email:emily.hall@ntu.ac.uk 


\section{Investigating factors affecting the body temperature of dogs competing in cross country (canicross) races in the $\mathrm{UK}$.}

\section{Abstract}

Increasing numbers of people are running with their dogs, particularly in harness through the sport canicross. Whilst canicross races are typically held in the winter months, some human centred events are encouraging running with dogs in summer months, potentially putting dogs at risk of heat related injuries, including heatstroke. The aim of this project was to investigate the effects of ambient conditions and running speed on post-race temperature of canicross dogs in the UK, and investigate the potential risk of heatstroke to canicross racing dogs. The effects of canine characteristics (e.g. gender, coat colour) were explored in order to identify factors that could increase the risk of exercise-induced hyperthermia (defined as body temperature exceeding the upper normal limit of $\left.38.8^{\circ} \mathrm{C}\right) .108$ dogs were recruited from 10 race days, where ambient conditions ranged from $-5-11^{\circ} \mathrm{C}$ measured as universal thermal comfort index (UTCI). 281 post race tympanic membrane temperatures were recorded, ranging from $37.0-42.5^{\circ} \mathrm{C}$. There was a weak correlation between speed and post-race temperature $(r=0.269, \mathrm{P}<0.001)$. Whilst no correlation between any single environmental factor or UTCI and post-race temperature was found, the proportion of dogs developing exercise-induced hyperthermia during the race increased with UTCI $(r=0.688, \mathrm{P}=0.028)$. Male dogs $(\chi(1)=18.286, \mathrm{P}<0.001)$, and dark coated dogs $(\chi(2)=8.234, \mathrm{P}=0.014)$, were significantly more likely to finish the race with a temperature exceeding $40.6^{\circ} \mathrm{C}$. Prolonged elevation of body temperature above this temperature is likely to cause heatstroke. At every race dogs exceeded this critical temperature, with $10.7 \%(n=30)$ of the overall study population exceeding this temperature throughout the study period. The results suggest male dogs, dark coloured dogs, and increased speed of running all increase the risk of heatstroke in racing canicross dogs. Further research is required to investigate the impact of environmental conditions on post-race cooling, to better understand safe running conditions for dogs.

Highlights

- Canicross races are typically run over winter to avoid running dogs in hot weather.

- Ambient temperature alone did not correlate with dogs' post-race temperatures.

- Average race speed had a positive effect on canine post-race temperature.

- Dogs exceeding heatstroke associated temperatures were mainly male with dark coats.

Keywords 
Exercise-induced hyperthermia, canine heatstroke, running with dogs, aural thermometer, canine athlete

\section{ABBREVIATIONS FOR FOOTNOTE}

TMT - tympanic membrane temperature

UTCI - universal thermal comfort index

\section{Introduction}

The sport canicross involves competitors completing a cross country style race either running, cycling or scootering, whilst harnessed to dogs (see figure 1). The dogs normally run ahead, taking up the slack in the bungee line and providing some assistance to the runner. Canicross is an effective means of exercising both runner and dog over relatively short 'sprint' distances of approximately $5 \mathrm{~km}$ (although longer competitive distances are run). Now formally recognised by The Kennel Club, the sport has been run competitively in the UK since 2000, with increasing numbers of competitors taking part in races around the country (The Kennel Club, 2017). As race results are ultimately linked to both human and dog speed, the sport can promote increased physical activity, encouraging people to exercise with their dog to improve their race times, competitive performance, health and fitness in both species.

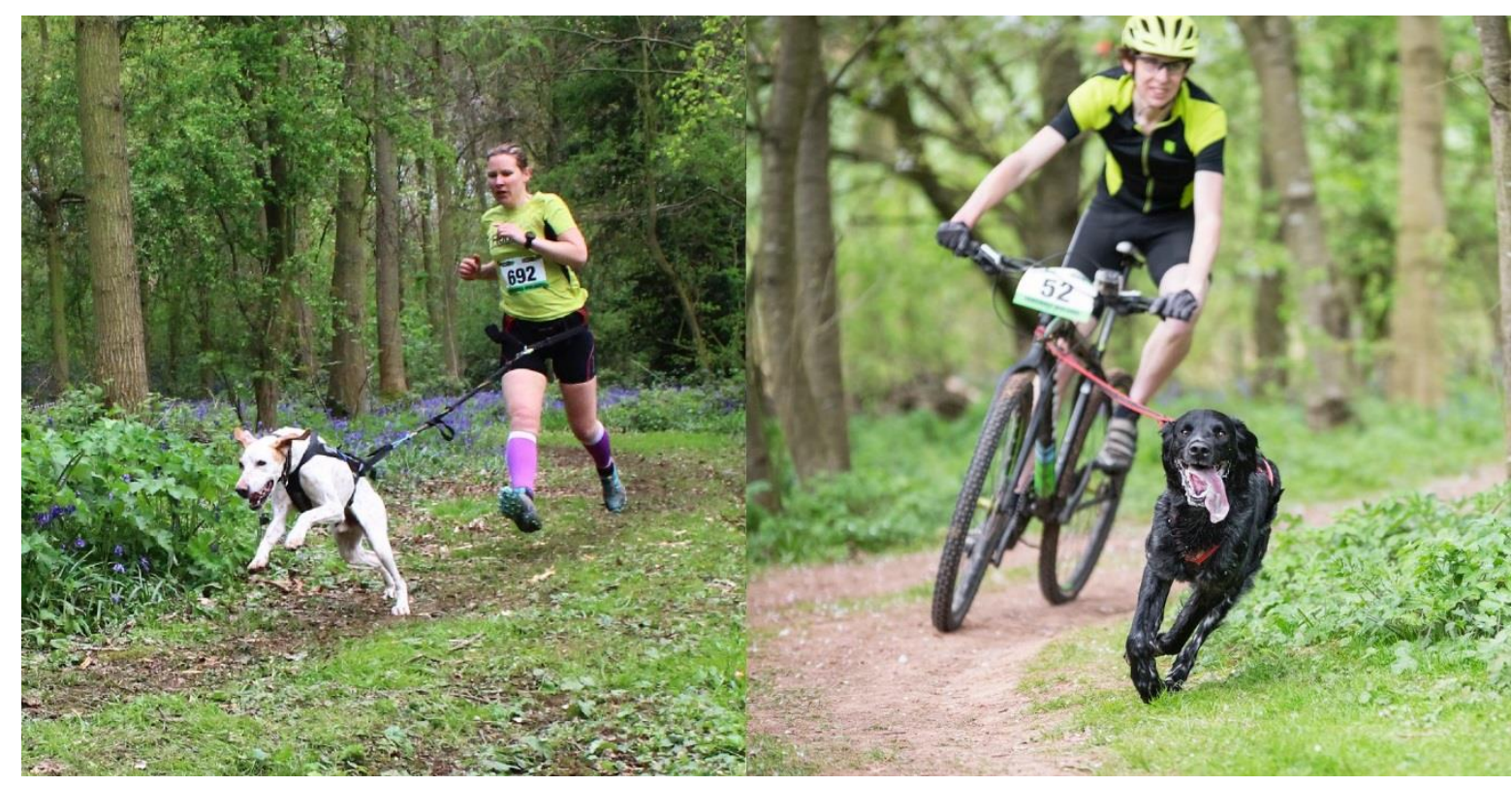

Figure 1: One dog canicross competitor on the left, one dog bikejor competitor on the right. 
There have been several studies exploring ways of encouraging dog owners to spend more time walking and exercising with their dogs (Rhodes et al., 2012; Schneider et al., 2014; Westgarth et al., 2014; Westgarth et al., 2015), however studies exploring the impact of this advice (both positive and negative) on the dog are lacking. Encouraging owners to increase their activity levels through dog walking or running, could place the dog at risk of conditions such as heatstroke, as unfit dogs show significantly reduced exercise endurance and increased rate of temperature rise (Nazar et al., 1992) compared to healthy dogs. As heatstroke is a potentially fatal condition, and has been reported following just six minutes of exercise in hot ambient conditions (Bruchim et al., 2006), advising owners to start exercising with their unfit dog in spring or summer months could prove extremely dangerous for the dog. At present advice regarding safe ambient temperature thresholds for exercising with dogs is lacking.

Heatstroke is defined as a systemic inflammatory response leading to multi organ dysfunction and brain damage, associated with hyperthermia (Bouchama and Knochel, 2002), in dogs heatstroke is typically associated with rectal temperatures exceeding $41^{\circ} \mathrm{C}$ (Flournoy et al., 2003). One veterinary hospital has reported an increase in the number of dogs presenting with exertional heatstroke (caused by exercise) compared to environmental heatstroke (typically following vehicle confinement), with $73 \%$ of recent cases being categorised as exertional heatstroke (Bruchim et al., 2017), compared to 58\% of cases previously reported (Aroch et al., 2009). The increase in popularity of amateur canine sports participation in the UK, combined with increasing episodes of warm weather during traditionally colder months in autumn and early spring (WMO, 2017), could potentially increase the risk of canine exertional heatstroke occurring (Hall and Carter, 2016).

Traditionally canicross races are run during the autumn-spring, to avoid warm weather for both the runners' and the dogs' benefit. Dogs are more likely to develop heatstroke following prolonged exercise in warm conditions, as they can only sweat through their paw pads, relying mainly on convection and radiation of heat, then panting to allow evaporative heat loss for thermoregulation (Johnson et al., 2006). As ambient temperature increases, heat loss through convection and radiation is limited (Johnson et al., 2006). When competing at canicross events owners are reminded to monitor their own dogs for signs of over-heating, and many canicross clubs have their own informal rules on safe competition conditions. Guidelines on suitable working temperatures are limited, and are reliant on personal experience and anecdote. One such recommendation used by canicross groups and on-line discussion forums is "do not run your dog if ambient temperature $\left({ }^{\circ} \mathrm{C}\right) \mathrm{x}$ humidity $(\%)>$ 
1000 ", where multiplying the ambient temperature by the relative humidity is used to determine if it is safe to run with your dog (Cani-Sports Edinburgh, ND; Highland Canicrossers, ND). Studies investigating the validity of this guideline are lacking, and to date, there have been no studies investigating body temperature in pet dogs competing in canicross races. Whilst the effect of exercise on body temperature has been investigated in both long distance sled racing dogs (Phillips et al., 1981), and in greyhounds competing in shorter sprint races under $1 \mathrm{~km}$ (McNicholl et al., 2016) there has been no research investigating the temperature of pet dogs racing over middle distances such a canicross race (around 3-5km),

To develop more robust guidelines for safe environmental conditions for canine sports, additional investigation into the effect of ambient conditions on canine athlete body temperature is needed. Physical exercise can exceed thermoregulatory mechanisms, potentially impacting animal health and performance (Piccone et al., 2012, Robbins et al., 2017). Monitoring body temperature is therefore important to monitor the health, physiological status and welfare of exercising, competing or working animals (Rizzo et al., 2017). As temperature, humidity and wind speed all influence body temperature, it is important to consider the thermal impact of the combined effect of all three, when investigating environmental impact on body temperature. Universal thermal comfort index (UTCI) incorporates all of these factors to calculate a "feels like" temperature that reflects the ambient conditions as a whole (Jenditzy et al., 2012). This allows individual environmental conditions to be measured in the field, then combined using the UTCI calculation to provide an ambient temperature that reflects the overall impact of the conditions present.

Aural thermometers measuring tympanic membrane temperature (TMT) have been investigated in comparison with rectal thermometers, and have been found to be an effective alternative for monitoring temperature in dogs pre- and post-exercise (Hall and Carter, 2017a; Robbins et al., 2017; Zanghi, 2016). As aural thermometers are faster and often better tolerated that rectal thermometers (Gomart et al., 2014; Lamb and McBrearty, 2013) they offer an ideal means of monitoring immediate post-race body temperature in the canine athlete under field conditions. Aural thermometers under-report body temperature when compared to rectal temperature in dogs, by around $0.4^{\circ} \mathrm{C}$ when measured with an animal specific device (Gomart et al., 2014; Hall and Carter, 2017a; Zanghi, 2016), and by around $1.3^{\circ} \mathrm{C}$ using a human aural thermometer (Piccone et al., 2011). It is therefore important to use 
an animal specific thermometer and an appropriate reference range when interpreting ear temperature readings (Hall and Carter, 2017b).

The aims of the study were to investigate the effects of varying ambient conditions on the tympanic membrane temperature (TMT) of privately owned pet dogs competing in middle distance canicross races, and the incidence of post-race temperatures associated with heatstroke. In addition, the effects of race speed, gender and coat colour on post-race temperature were also explored to identify canine characteristics that could increase the risk of exercise-induced hyperthermia.

\section{Methods}

This study was approved by Nottingham Trent University's School of Animal, Rural and Environmental Sciences ethics committee.

\subsection{The race courses}

Canicross runners, scooter and bikejor competitors competed with their dogs over a course $3.8-4.5 \mathrm{~km}$ in length over two consecutive days (the course was identical on both days). All dogs had previously competed in canicross races and were at least one year of age for canicross, and two years for scooter and bikejor races. Data were collected at five race weekends (10 individual races) over the 2015-16 and 2016-17 Canicross Midlands race seasons, run between November and April at four venues in the East and West Midlands, UK.

\subsection{The animals}

Canine participants were recruited opportunistically from those competing at the canicross events. Between 18 and 35 dogs were examined on each race day, providing data for 108 dogs (59 male, 49 female) aged 1-10 years old (mean 4.4 years) over the 10 races. Breed types represented were: pointer $(n=31)$, collie $(n=22)$, spaniel $(n=13)$, husky $(n=10)$, retriever $(n=8)$ and other $(n=24)$. Across the multiple race dates, dogs' temperatures were recorded pre and post-race between one and nine times (mean 2.6 times per dog) to give 281 post-race temperature data points, of which 210 also had pre-race temperature recorded. For the purpose of analysis, each of the dog's race dates were treated as a separate data point.

\subsection{Temperature Measurements}


TMT was measured pre-race in each dog in the two-hour period prior to competition and post-race, immediately after crossing the finish line of the race. As sampling was opportunistic it was not possible to obtain pre-race TMT readings for all dogs. Left or right ears were selected depending on the positioning of the dog following light restraint. TMT was recorded using a Vet-Temp VT-150 Instant Ear Thermometer (Advanced Monitors Corporation, California, USA), covered by a single use Vet-Temp DPC-500 probe cover. The Vet-Temp thermometer measures temperatures between $32.2^{\circ} \mathrm{C}$ and $43.3^{\circ} \mathrm{C}$, with an accuracy of $\pm 0.2^{\circ} \mathrm{C}$. The thermometer was used as per the manufacturer's instructions with no lubrication, and a reading being obtained following the audible alarm. If a reading reported an error code, the probe cover was changed, and the process repeated. If an error code occurred a second time, the reading was discounted.

Hyperthermia was defined as TMT greater than the upper normal limit of $38.8^{\circ} \mathrm{C}$, using the previously established normal canine TMT range of $36.8-38.8^{\circ} \mathrm{C}$ (Hall and Carter, 2017b). The number of dogs' temperatures exceeding $40.6^{\circ} \mathrm{C}$ was also noted, as this is equivalent to a rectal temperature of $41^{\circ} \mathrm{C}$ considered to be the critical body temperature over which heatstroke is likely; TMT has been shown to measure approximately $0.4^{\circ} \mathrm{C}$ lower than rectal temperature following exercise (Hall and Carter, 2017a; Zanghi, 2016).

\subsection{Ambient conditions}

Ambient conditions (temperature, humidity and wind speed) were recorded prior to collecting the pre-race TMT and at approximately 30-minute intervals until the last post-race temperature had been recorded. Measurements were taken using a HI 9564 Thermo Hygrometer (Hanna Instruments Lid, Bedfordshire, UK), and RD 506-9650 Anemometer (R.S. Components Ltd., Northamptonshire, UK). To evaluate the overall impact of these environmental conditions, the results were used to calculate Universal Thermal Comfort Index (UTCI) values (Jendritzy et al., 2012). UTCI was calculated from ambient temperature $\left({ }^{\circ} \mathrm{C}\right)$, relative humidity $(\%)$ and wind speed $(\mathrm{m} / \mathrm{s})$ using the UTCI calculator (http://www.utci.org/). The mean UTCI temperature for the duration of the race period was then calculated. Additionally, the mean 'ambient temperature $\mathrm{x}$ humidity' was calculated for each race event.

The shade temperature recorded at the nearest Met Office weather station was also recorded for the duration of the race, to allow comparison between this and the non-shade on-site temperature measurement. 


\subsection{Additional information}

Time to complete the course was used to calculate average race speed $(\mathrm{km} / \mathrm{h})$, age of the $\mathrm{dog}$, breed, coat colour and coat length and were recorded for all participants.

\subsection{Statistical analysis}

Data were checked for normality using Kolmogorov-Smirnov tests and statistics were calculated using SPSS 23.0 (SPSS Inc., Chicago, IL). The study was a within subject design to accommodate for the range of breeds that participated in each location. This ensured that individual baseline temperature differences were accounted for. Paired t-tests were used to determine the effect of gender on pre-race temp, post-race TMT and temperature difference. ANOVA and post hoc (Bonferroni) tests were used to assess the effect of coat colour and coat length on pre-race TMT, post-race TMT and temperature difference.

To determine the effects of UTCI and speed on post-race temperature, a general linear model (GLM) was carried out, with gender included as a fixed effect and UTCI and speed included as covariates. This GLM was rerun using TMT increase to determine the effects of UTCI and speed on TMT increase. A GLM was also used to look at impact of separate ambient conditions on post-race TMT, with gender as a fixed effect and ambient temperature, humidity and windspeed were included as covariates. This GLM was rerun using TMT increase to determine the effects of separate ambient conditions on TMT increase.

In addition, to investigate the effect of 'ambient temperature $x$ humidity' values, a GLM was run on post-race TMT, with gender as a fixed effect, and speed and 'ambient temperature $\mathrm{x}$ humidity' values as covariates. This GLM was rerun using TMT increase.

Chi squared was used to look at the association between gender, coat length and coat colour respectively on the proportion of dogs with post-race TMT $>38.8^{\circ} \mathrm{C}$ then $>40.6^{\circ} \mathrm{C}$. Pearson's correlations were used to determine the relationships between the proportion of dogs reaching post-race TMT $>38.8^{\circ} \mathrm{C}$ and race ambient temperature, humidity, wind speed or 'ambient temperature x humidity'. Pearson's correlations were calculated to determine the relationship between the proportion of dogs with a post-race TMT $>40.6{ }^{\circ} \mathrm{C}$ and UTCI, and the proportion of dogs with a post-race TMT $>40.6^{\circ} \mathrm{C}$ and 'ambient temperature $\mathrm{x}$ humidity' value. 


\section{Results}

\subsection{Ambient conditions}

Across the 10 race days the mean ambient temperature recorded was $8.8^{\circ} \mathrm{C}$ (range 3.3 $15.4^{\circ} \mathrm{C}$ ), the mean shade temperature recorded by the Met Office weather station was $7.5^{\circ} \mathrm{C}$ (range $2.5-13.5^{\circ} \mathrm{C}$ ) with the Met Office temperature recording a lower temperature on all but one occasion and reading up to $5.5^{\circ} \mathrm{C}$ lower than the site temperature. Mean site humidity was $70.3 \%$ (range $55.0-96.1 \%$ ), mean site wind speed was $1.7 \mathrm{~m} / \mathrm{s}$ (range $0-7.4 \mathrm{~m} / \mathrm{s}$ ). The mean calculated UTCI temperature for all race days was $7.3^{\circ} \mathrm{C}$ (range $-5.0-11.7^{\circ} \mathrm{C}$ ).

\subsection{Effect of canine characteristics on tympanic membrane temperature}

The pre-race TMT varied by $3.1^{\circ} \mathrm{C}$ across all readings, and was consistent across gender $(\mathrm{Z}=$ -0.527, $\mathrm{P}=0.598)$, coat colour $(\mathrm{F}=0.176, \mathrm{P}=0.839)$ and coat length $(\mathrm{F}=1.042, \mathrm{P}=0.355)$. Male dogs had a significantly greater TMT increase from pre to post-race than female dogs $(\mathrm{Z}=-4.510, \mathrm{P}<0.001)$. There was no effect of coat colour $(\mathrm{F}=1.743, \mathrm{P}=0.178)$ or coat length $(\mathrm{F}=1.713, \mathrm{P}=0.183)$ on TMT increase (see table 1$)$.

Table 1: Descriptive statistics of pre-race tympanic membrane temperature and temperature increase, for each category of dog.

\begin{tabular}{|l|l|l|}
\hline Study population & $\begin{array}{l}\text { Mean (range) pre-race } \\
\text { temperature } \\
\left({ }^{\circ} \mathrm{C}\right)\end{array}$ & $\begin{array}{l}\text { Mean (range) temperature } \\
\text { increase } \\
\left({ }^{\circ} \mathrm{C}\right)\end{array}$ \\
\hline All dogs $(\mathrm{n}=210)$ & $37.9(36.2-39.1)$ & $1.8(-1.4-5.0)$ \\
\hline Male $(\mathrm{n}=113)$ & $37.7(36.2-39.0)$ & $2.1(-0.4-4.1)^{\mathrm{a}}$ \\
\hline Female $(\mathrm{n}=97)$ & $37.7(36.3-39.1)$ & $1.4(-1.4-5.0)$ \\
\hline Dark coat colour $(\mathrm{n}=124)$ & $37.7(36.2-39.1)$ & $1.8(-0.9-5.0)$ \\
\hline Medium coat colour $(\mathrm{n}=34)$ & $37.7(36.3-39.0)$ & $1.5(-0.1-3.4)$ \\
\hline Pale coat colour $(\mathrm{n}=52)$ & $37.6(36.2-38.9)$ & $1.9(-1.4-3.8)$ \\
\hline Short coat $(\mathrm{n}=105)$ & $37.6(36.3-39.0)$ & $1.7(-0.9-4.9)$ \\
\hline Medium coat $(\mathrm{n}=101)$ & $37.8(36.2-39.1)$ & $1.8(-1.4-5.0)$ \\
\hline Long coat $(\mathrm{n}=4)$ & $37.6(37.2-38.3)$ & $2.7(2.0-3.6)$ \\
\hline
\end{tabular}

Significances $(P<0.05)$ : (effect of gender $)^{a}$ vs female 
The post-race TMT varied by $5.2^{\circ} \mathrm{C}$ across all readings, with male dogs getting significantly hotter than female dogs $(Z=-4.048, P<0.001)$ (see table 2$)$. Coat colour also affected postrace TMT $(F=4.312, P=0.014)$, post hoc tests using the Bonferroni correction revealed that dogs with medium colour coats had lower post-race TMT than dark coated dogs, they also showed a tendency towards a lower TMT than dogs with pale coats. No difference was seen between dark and pale coats on TMT. Coat length did not affect post-race temperature $(\mathrm{F}=$ 2.206, $\mathrm{P}=0.112)$.

Table 2: Descriptive statistics of post-race tympanic membrane temperature, for each category of dog.

\begin{tabular}{|l|l|}
\hline Study population & $\begin{array}{l}\text { Mean (range) pre-race temperature } \\
\left({ }^{\circ} \mathrm{C}\right)\end{array}$ \\
\hline All dogs $(\mathrm{n}=281)$ & $39.4(37.0-42.5)$ \\
\hline Male $(\mathrm{n}=157,55.9 \%)$ & $39.6(37.6-42.5)^{\mathrm{a}}$ \\
\hline Female $(\mathrm{n}=124,44.1 \%)$ & $39.1(37.0-42.4)$ \\
\hline Dark coat colour $(\mathrm{n}=161,57.3 \%)$ & $39.5(3701-42.5)$ \\
\hline Medium coat colour $(\mathrm{n}=48,17.1 \%)$ & $39.0(37.4-41.1)^{\mathrm{b}}$ \\
\hline Pale coat colour $(\mathrm{n}=72,25.6 \%)$ & $39.5(37.0-40.9)$ \\
\hline Short coat $(\mathrm{n}=135,48.0 \%)$ & $39.3(37.1-42.5)$ \\
\hline Medium coat $(\mathrm{n}=130,46.3 \%)$ & $39.5(37.0-42.5)$ \\
\hline Long coat $(\mathrm{n}=16,5.7 \%)$ & $39.6(37.5-41.9)$ \\
\hline
\end{tabular}

Significances $(P<0.05)$ : (effect of gender $)^{a}$ vs female, (effect of coat colour $)^{b}$ vs dark

\subsection{Effects of speed and ambient conditions on tympanic membrane temperature}

The average speed $($ mean $=15.58 \mathrm{~km} / \mathrm{h}$, range $7.82-28.58 \mathrm{~km} / \mathrm{h}$ ) of the dog during the race had a significant effect on the post-race TMT $(\mathrm{F}=7.729, \mathrm{P}=0.006)$ but no effect on TMT increase $(\mathrm{F}=2.843, \mathrm{P}=0.093)$. Speed was found to have a weak correlation with post-race TMT $(r=0.269, n=279, P<0.001)$. UTCI had no effect on either post-race TMT $(\mathrm{F}=0.478$, 
$\mathrm{P}=0.490)$ or TMT increase $(\mathrm{F}=0.137, \mathrm{P}=0.712)$. Individual environmental conditions of temperature, humidity, wind speed and 'ambient temperature $\mathrm{x}$ humidity' values had no effect on either post-race TMT or TMT increase $(\mathrm{P}>0.05)$.

\subsection{Critical temperatures}

The proportion of dogs reaching post-race TMT $>38.8^{\circ} \mathrm{C}$ was calculated for each individual race day. As race day average UTCI temperature increased, the proportion of dogs reaching post-race $\mathrm{TMT}>38.8^{\circ} \mathrm{C}$ increased $(\mathrm{r}=0.688, \mathrm{n}=10, \mathrm{P}=0.028)$ (see figure 2$)$. However, there was no correlation between proportion of dogs reaching post-race TMT $>38.8^{\circ} \mathrm{C}$ and race ambient temperature $(\mathrm{r}=0.356, \mathrm{n}=10, \mathrm{P}=0.312)$, humidity $(\mathrm{r}=-0.204, \mathrm{P}=0.571)$ or wind speed $(\mathrm{r}=-0.512, \mathrm{n}=10, \mathrm{P}=0.131)$. In addition, no correlation occurred between proportion of dogs reaching post-race TMT $>38.8^{\circ} \mathrm{C}$ and 'ambient temperature $\mathrm{x}$ humidity' $(\mathrm{r}=0.194, \mathrm{n}=10, \mathrm{P}=0.592)$.

Figure 2: Scatter plot showing race universal thermal comfort index temperature against the proportion of dogs developing post-race hyperthermia (temperature $>38.8^{\circ} \mathrm{C}$ ). The theoretical point at which all dogs would develop post-race hyperthermia is $22.0^{\circ} \mathrm{C}$.

No association was found between gender $(\chi(1)=0.386, \mathrm{P}=0.224)$, or coat length $(\chi(2)=$ $0.222, \mathrm{P}=0.217$ ) and proportion of dogs with post-race $\mathrm{TMT}>38.8^{\circ} \mathrm{C}$. Fewer medium colour and more pale dogs that expected had a post-race $\mathrm{TMT}>38.8^{\circ} \mathrm{C}(\chi(2)=9.196, \mathrm{P}=$ $0.010)$.

The dogs reaching the critical temperature of greater than $40.6^{\circ} \mathrm{C}$, comprised the top $10.7 \%$ of all post-race TMT readings recorded across the 10 race days $(n=30,24$ male dogs and six female dogs). More male dogs $(80 \%)$ were present than expected $(\chi(1)=18.286, \mathrm{P}<0.001)$. There was no correlation between proportion of dogs reaching post-race TMT $>40.6^{\circ} \mathrm{C}$ and race UTCI ( $\mathrm{r}=0.221, \mathrm{n}=10, \mathrm{P}=0.539)$. Additionally, no correlation occurred between proportion of dogs reaching post-race TMT $>40.6^{\circ} \mathrm{C}$ and 'ambient temperature $\mathrm{x}$ humidity' $(\mathrm{r}=-0.063, \mathrm{n}=10, \mathrm{P}=0.862)$. No association was found between coat length and proportion of dogs exceeding the critical temperature $40.6{ }^{\circ} \mathrm{C}(\chi(2)=2.571, \mathrm{P}=0.091)$. More dark coated dogs (76.7\%) and fewer pale coated dogs (13.3\%) were found to exceed post-race TMT $>40.6^{\circ} \mathrm{C}$ than expected $(\chi(2)=8.234, \mathrm{P}<0.05)$.

\section{Discussion}


This study found no effect of ambient temperature alone on post-race TMT in dogs exercising in ambient conditions ranging from $-5.0-11.7^{\circ} \mathrm{C}$ (UTCI), which is in line with a previously published study investigating strenuous exercise tests on Labradors (Matwichuk et al., 1999). In contrast, McNicholl et al. (2016) found a small effect of ambient temperature on greyhound post-race temperatures, and Phillips et al. (1981) found a strong effect of ambient temperature on mean post-race rectal temperature in sled dogs. These conflicting findings, could be explained by differences in canine management between these four studies. The greyhounds and sled dogs were all managed by professional dog trainers, so would have been prepared for each of their races in a similar manner to ensure performance, with similar feeding, hydration and specific conditioning for the type of work being undertaken. In contrast, the dogs in this study, and the Labradors undergoing exercise tests, were all privately owned pet dogs likely managed in very different ways.

The body temperature of exercising dogs is known to be influenced by many nonenvironmental factors. The results of this study support the findings of McNicholl et al. (2016); male dogs were significantly hotter post-race compared to female dogs, dark dogs developed significantly higher temperatures post-race compared to medium coloured dogs (but not pale dogs), and dog's exceeding the post-race temperature of $40.6^{\circ} \mathrm{C}$ were more likely to be male, and dark coated. This study also supports the findings of Chapman and Baker (1984), increasing exercise intensity - in this case speed - had a positive effect on postrace temperature. Body size (McNicholl et al., 2016; Phillips et al., 1981), conditioning (Ferasin and Marcora, 2009; Nazar et al., 1992; Ready and Morgan, 1984), hydration (Baker et al., 1983) and diet (Ober et al., 2016) have all be found to influence post exercise body temperature. Although these factors were not investigated as part of this project, they are likely to have influenced the results. It is probable that the pet dogs taking part in this study, would have experienced variations in their feeding, hydration and conditioning both between different dogs, and between races for individual dogs. Additionally, the different race courses had different terrains and inclines at different points in the race, also known to influence rate of temperature elevation in exercising dogs (Chapman and Baker, 1984). The combination of these varying factors could have influenced both rate of temperature increase, and post-race temperature and may explain the lack of correlation between UTCI temperature, and post-race temperature of the dogs.

Measuring a single environmental parameter cannot represent the true thermal environment experienced by a human, this lead to development of the UTCI calculation (Jendritzy et al., 
2012). It is therefore unsurprising there were no correlations between either post-race TMT, or proportion of dogs developing exercise-induced hyperthermia post-race, and any one environmental condition - wind speed, humidity or temperature - or even the combination of temperature and humidity. Whilst no direct correlation was found between UTCI temperature and post-race canine TMT, there was a significant correlation between UTCI and the proportion of dogs finishing the race with exercise-induced hyperthermia. Extrapolating the "line of best fit" in figure 2, theoretically all racing dogs would develop hyperthermia once the UTCI temperature reaches $22.0^{\circ} \mathrm{C}$. Whilst hyperthermia in itself is not dangerous, prolonged hyperthermia particularly above $41^{\circ} \mathrm{C}$ increases the likelihood of long-term damage from heatstroke.

At every race in this study, at least one dog developed a post-race body temperature that would be considered at risk for developing heatstroke (TMT $>40.6^{\circ} \mathrm{C}$ ). The highest recorded post-race TMT was $42.5^{\circ} \mathrm{C}$. This is comparable to the highest TMT $\left(42.4^{\circ} \mathrm{C}\right)$ reported by Robbins et al. (2017), following 30 minutes of intermittent exercise in ambient conditions of $28.7^{\circ} \mathrm{C}$ and $49.6 \%$ humidity. This highlights the relative intensity of canicross races; a 12-30 minute race in relatively cool ambient conditions, caused body temperature to elevate to a similar level recorded in dogs exercising for 30 minutes in ambient conditions approaching body temperature. Despite these body temperature elevations, no dogs exhibited any clinical symptoms of heatstroke during any of the canicross race days, again mirroring the findings of Robbins et al. (2017). On several occasions, owners requested additional temperature checks on particularly hot dogs during the period following the race. These dogs all returned to a normal body temperature within 10-20 minutes, suggesting appropriate cooling mechanisms were in place to prevent prolonged hyperthermia. As ambient temperature increases, particularly as it approaches body temperature, cooling mechanisms become less effective (Hemmelgarn and Gannon, 2013). Further investigation into the factors affecting post exercise cooling, particularly the impact ambient conditions have on rate of cooling, may be more required to establish guidance for safe ambient conditions for running.

The combination of canine factors, husbandry factors, exercise factors and environmental factors all influence canine post exercise temperature, meaning any guidelines for "safe" running temperatures are unlikely to apply to all animals and could potentially put dogs at risk. Instead, owners should be encouraged to understand the factors that can influence their own dog's performance and heat tolerance in different ambient conditions. Canicross race organisers, and any other organisations promoting running with dogs, should be aware that 
the recommendation to not run a dog if 'ambient temperature $\left({ }^{\circ} \mathrm{C}\right) \mathrm{x}$ humidity $(\%)>1000^{\prime}$ ' did not correlate to canine body temperature or the proportion of dog's developing hyperthermia, or the proportion of dogs developing a temperature considered at risk of heatstroke, so its continued use cannot be recommended for the general pet dog population. As local weather stations only provide estimates of local shade temperatures, this could promote a false sense of security regarding race conditions. Instead, race organisers should make efforts to provide on-site means of monitoring environmental conditions, including ambient temperature, humidity and wind speed, preferably providing an estimation of UTCI temperature, for instance using a wet bulb globe heat stress monitor. Being able to measure ambient conditions on the race course itself, allows owners to make an informed decision regarding their animal's ability to compete under those conditions. Dog owners need to be aware of the factors that can impact their animal's exercising body temperature. An unfit, poorly acclimatised, dark coated male dog may be at greater risk of heatstroke running in late autumn, than a pale coated, female dog in regular training, running in the middle of summer.

A major limitation of this study, was the inability to measure body temperature during the race. Previous work using ingestible telemetric thermometry capsules in Labradors, has shown that body temperature increased continuously during a $3.5 \mathrm{~km}$, flat run (Angle and Gillette, 2011). One study using treadmill running dogs, suggested that reaching a peak body temperature coincided with the point of exhaustion for the dogs (Nazar et al., 1992), with unconditioned dogs reaching peak temperature and therefore exhaustion faster than conditioned dogs. Phillips et al., (1981) continuously measured rectal temperature in racing sled dogs over a $16 \mathrm{~km}$ run. They found temperature increased up to around 25 minutes into the run, then tended to plateau following several short rest stops triggered by the trainer's perception of the dog's ability to continue.

As intensity of exercise, specifically incline, has been shown to significantly increase canine body temperature (Chapman and Baker, 1984), it is possible canicross racing dogs reach a peak body temperature following a period of incline work, which then plateaus or drops as the work intensity reduces. Anecdotally, canicross runners have reported their dogs slowing down and refusing to pull during races, potentially due to reaching their temperature tolerance threshold. As there are limited non-invasive methods of continuously measuring core body temperature in dogs available in the UK, it is currently difficult to conduct further research on this area under field conditions using pet owned dogs. However, understanding how the dog's body temperature changes throughout the race in response to speed, inclines 
and the presence of water obstacles on the course, may help to suggest appropriate race modifications to prevent canine heatstroke, when ambient conditions are considered too warm for all dogs to safely compete.

\section{Conclusion}

This study found no direct correlation between ambient conditions or UTCI temperature and post-race body temperature of dogs competing in canicross races, and at every race dogs reached post-race temperatures exceeding the heatstroke risk threshold $\left(40.6^{\circ} \mathrm{C}\right)$. Average race speed was found to have a positive effect on post-race temperature, and a positive correlation between UTCI $\left({ }^{\circ} \mathrm{C}\right)$ and the proportion of dogs developing exercise-induced hyperthermia during the race was also found. The lack of a clear relationship between environmental factors and canine body temperature highlights the difficulty of trying to establish guidelines for safe running temperatures for dogs. Canine sports associations, and dog owners in general should be aware of the potential for inter and intra-dog temperature variations, depending on both husbandry and canine factors, and should be aware of the risk of heatstroke even on relatively cold UK winter days.

\section{Acknowledgements}

The authors would like to thank the committee, members and dogs of Canicross Midlands for their on-going support, and willingness to participate in this project. Additional thanks go to Dr Jaime Martin, for his support in the statistical analysis of this study, Becky Harding and Jackie Burrell for images supplied.

This research did not receive any specific grant from funding agencies in the public, commercial, or not-for-profit sectors.

\section{References}

Angle, T.C. and Gillette, R.L., 2011. Telemetric measurement of body core temperature in exercising unconditioned Labrador retrievers. Can. J. Vet. Res. 75, 157-159.

Aroch, I., Segev, G., Loeb, E., and Bruchim, Y. 2009. Peripheral nucleated red blood cells as a prognostic indicator in heatstroke in dogs. J. Vet. Int. Med. 23, 544-51.

DOI:10.1111/j.1939-1676.2009.0305.x 
Baker, M.A., Doris, P.A. and Hawkins, M.J., 1983. Effect of dehydration and hyperosmolality on thermoregulatory water losses in exercising dogs. Am. J. Physiol. 244, R516-R521.

Bouchama, A. and Knochel, J.P., 2002. Heat stroke. N. Eng. J. Med. 346, 1978-1988.

Bruchim, Y., Klement, E., Saragusty, J., Finkeilstein, E., Kass, P., and Aroch, I., 2006. Heat stroke in dogs: A retrospective study of 54 cases (1999-2004) and analysis of risk factors for death. J. Vet. Int. Med, 20, 38-46. DOI:10.1111/j.1939-1676.2006.tb02821.x

Bruchim, Y., Kelmer, E., Cohen, A., Codner, C., Segev, G. and Aroch, I., 2017. Hemostatic abnormalities in dogs with naturally occurring heatstroke. J. Vet. Emerg. Crit. Care 27, 315324. DOI: $10.1111 /$ vec. 12590

Cani-Sports Edinburgh, no date. Frequently Asked Questions.

http://canisportsedinburgh.co.uk/?page_id=15 (accessed 26.07.17)

Chapman, L.W. and Baker, M.A., 1984. Cardiac output of dogs exercising in the heat. Am. J. Physiol. 247, R124-R12.

Ferasin, L. and Marcora, S., 2009. Reliability of an incremental exercise test to evaluate acute blood lactate, heart rate and body temperature responses in Labrador retrievers. J. Comp. Physiol. B, 179(7), pp.839-845. DOI: 10.1007/s00360-009-0367-z

Flournoy, S., Wohl, J. and Macintire, D.K., 2003. Heatstroke in dogs: pathophysiology and predisposing factors. Comp. Cont. Educ. 25, 410-417.

Gomart, S.B., Allerton, F.J.W. and Gommeren, K., 2014. Accuracy of different temperature reading techniques and associated stress response in hospitalised dogs. J. Vet. Emerg. Crit. Care 24: 279-285. DOI:10.1111/vec.12155

Hall, E.J. and Carter, A., 2016. Heatstroke - providing evidence-based advice to dog owners. Vet. Nurs. J. 31, 359-363. DOI: 10.1080/17415349.2016.1245119

Hall, E.J. and Carter, A.J., 2017a. Comparison of rectal and tympanic membrane temperature in healthy exercising dogs. Comp. Exerc. Physiol. 13, 37-44. DOI: 10.3920/CEP160034 Hall, E.J. and Carter, A., 2017b. Establishing a reference range for normal canine tympanic membrane temperature. Vet. Nurs. J. 32, 369-373. DOI: 10.1080/17415349.2017.1377133 
Hemmelgarn, C. and Gannon, K. 2013. Heatstroke: Thermoregulation, Pathophysiology and Predisposing Factors. Compend. Contin. Educ. Pract. Vet. E1-E7.

Highland Canicrossers, no date. Highland Canicrossers JogScotland Session https://www.highlandcanicrossers.co.uk/?product=highland-canicrossers-jogscotland-session (accessed 26.07.17)

Jendritzy, G., De Dear, R. and Havenith, G., 2012. UTCI - Why another thermal index? Int. J. Biometeorol. 56, 421-428. DOI 10.1007/s00484-011-0513-7

Johnson, S.I., McMichael, M. and White, G., 2006. Heatstroke in small animal medicine: a clinical practice review. J. Vet. Emerg. Crit. Care, 16, 112-119.

DOI:10.1111/j.1476-4431.2006.00191.x

Lamb, V. and McBrearty, A.R., 2013. Comparison of rectal, tympanic membrane and axillary temperature measurement methods in dogs. Vet. Rec. 173, 524-528. DOI: 10.1136/vr.101806

Matwichuk, C.L., Taylor, S., Shmon, C.L., Kass, P.H. and Shelton, G.D., 1999. Changes in rectal temperature and hematologic, biochemical, blood gas, and acid-base values in healthy Labrador Retrievers before and after strenuous exercise. Am. J. Vet. Res. 60, 88-92.

McNicholl, J., Howarth, G.S. and Hazel, S.J., 2016. Influence of the environment on Body Temperature of racing greyhounds. Front. Vet. Sci. 3:53. DOI: 10.3389/fvets.2016.00053

Nazar, K., Greenload, J.E. and Pohoska, E., 1992. The effect of prolonged restriction of physical activity on exercise performance in dogs. Aviat. Space Environ. Med. 63, 684-688.

Ober, J., Gillette, R.L., Angle, T.C., Haney, P., Fletcher, D.J. and Wakshlag, J.J., 2016. The effects of varying concentrations of dietary protein and fat on blood gas, hematologic serum chemistry, and body temperature before and after exercise in Labrador retrievers. Front. Vet. Sci. 3, 59. DOI: 10.3389/fvets.2016.00059

Phillips, C.J., Coppinger, R.P. and Schimel, D.S., 1981. Hyperthermia in running sled dogs. J.Appl. Physiol. 51, 135-142.

Piccione, G., Giannetto, C., Fazio, F. and Giudice, E., 2011. Accuracy of auricular temperature determination as body temperature index and its daily rhythmicity in healthy dog. Biol. Rhythm Res., 42(5), pp.437-443. DOI: 10.1080/09291016.2010.526425 
Piccione, G., Casella, S., Panzera, M., Giannetto, C. and Fazio, F., 2012. Effect of moderate treadmill exercise on some physiological parameters in untrained beagle dogs. Exp. Anim. 61(5), pp.511-515. DOI: 10.1538/expanim.61.511

Ready, A.E. and Morgan, G., 1984. The physiological response of Siberian Husky dogs to exercise: effect of interval training. Can. Vet. J. 25 86-91.

Rhodes, R. E., Murray, H., Temple, V.A., Tuokko, H., Higgins, J.W., 2012. Pilot study of a dog walking randomized intervention: Effects of a focus on canine exercise. Prev. Med. 54, 309-312. DOI: 10.1016/j.ypmed.2012.02.014

Rizzo, M., Arfuso, F., Alberghina, D., Giudice, E., Gianesella, M. and Piccione, G., 2017. Monitoring changes in body surface temperature associated with treadmill exercise in dogs by use of infrared methodology. J. Therm. Biol. 69, pp.64-68. DOI: 10.1016/j.jtherbio.2017.06.007

Robbins, P.J., Ramos, M.T., Zanghi, B.M. and Otto, C.M., 2017. Environmental and physiological factors associated with stamina in dogs exercising in high ambient temperatures. Front. Vet. Sci. 4, p.144. DOI: 10.3389/fvets.2017.00144

Schneider, K.L., Murphy, D., Ferrara, C., Oleski, J., Panza, E.,; Savage, C., Gada, K., Bozzella, B., Olendzki, E., Kern, D. and Lemon, S.C., 2014. An online social network to increase walking in dog owners: A randomized trial. Med. Sci. Sports Exerc. 47, 631-639. DOI: 10.1249/MSS.0000000000000441

The Kennel Club, 2017. Canicross. http://www.thekennelclub.org.uk/activities/canicross/ (accessed 22.07.17)

Westgarth, C., Christley, R. and Christian, H., 2014. How might we increase physical activity through dog walking?: A comprehensive review of dog walking correlates. Int. J. Behav. Nutr. Phys. Act. 11, 83. https://doi.org/10.1186/1479-5868-11-83

Westgarth, C., Christian, H.E. and Christley, R.M., 2015. Factors associated with daily walking of dogs. BMC Vet. Res. 11, 116. DOI 10.1186/s12917-015-0434-5

WMO, 2017. High temperatures and extreme weather continue. https://public.wmo.int/en/media/news/high-temperatures-and-extreme-weather-continue (accessed 17.08.17) 
Zanghi, B.M., 2016. Eye and Ear Temperature Using Infrared Thermography are Related to Rectal Temperature in Dogs at Rest or With Exercise. Front. Vet. Sci. 3, 111.

DOI: $10.3389 /$ fvets.2016.00111 